\title{
Note
}

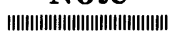

\section{Positron Emission Tomography Image Reconstruction Method Utilizing Magnetic Resonance Image ${ }^{\dagger}$}

\author{
Kenya Murase, Youpu ZhanG*, Yilong MA*, Koji Yamamoto** and \\ Alan C. Evans* \\ Department of Radiology, Ehime University School of Medicine \\ Shitsukawa, Shigenobu-cho, Onsen-gun, Ehime-Pref. 791-02, Japan \\ *McConnell Brain Imaging Center, Montreal Neurological Institute, McGill University \\ Montreal, Canada \\ **Department of Computer Science and Systems Engineering, Faculty of \\ Engineering, Miyazaki University \\ Gakuenkonohana-nishi, Miyazaki-shi, 889-21, Japan
}

Received October 7, 1996

\begin{abstract}
Magnetic resonance imaging (MRI) provides high-resolution anatomical information. In this study, we investigated the usefulness of an MRI-constrained method for image reconstruction of positron emission tomography (PET). We started the reconstruction process with a conventional Bayesian reconstruction (BAY) method. Typically after 7 iterations, we calculated the apparent radioactivity ratio between gray matter and other tissues such as white matter and cerebrospinal fluid from both the PET image and tissue classification probability images generated from segmented MR images, and then increased this ratio to enhance the functional boundary gradient. We multiplied the apparent ratio by 2.0. Subsequently, the cross-correlation between anatomical and enhanced functional boundary maps was incorporated into BAY using the weighted line site method. This algorithm was tested with simulated PET projection data generated from an MRI-based three-dimensional brain phantom, including the effects of resolution, attenuation, scatter and Poisson noise. The reconstructed images were evaluated in terms of root mean square distance (RMSD) from the true image. This method improved $R M S D$ by $39.6 \%, 33.5 \%$, and $33.0 \%$ on average, compared to ( $i$ ) the filtered backprojection method, (ii) the maximum-likelihood expectationmaximization method and (iii) BAY method, respectively. Our preliminary results suggest that incorporation of both anatomical and tissue classification information into the image reconstruction process is useful for improving the qualitative and quantitative accuracy of PET images.
\end{abstract}

Key Words: MRI, PET, Bayesian image reconstruction, anatomical information, tissue classification

\section{Introduction}

There are several methods for image reconstruction in positron emission tomography

†核磁気共鳴画像を利用したPET 画像再構成法。 村瀬研也, Youpu ZHANG*, Yilong MA*, 山本皓二**, Alan C. EvANS* : 愛媛大学医学部放射線医学教室, 791-02 愛媛県温泉郡重信町志津川, ${ }^{*}$ McConnell Brain Imaging Center, Montreal Neurological Institute, McGill University, Montreal, Canada, **宮崎大学工学部情報工学科情報処理システム講座, 889-21 宮崎市学園木花台西 1-1。
(PET). The most common method used in commercial PET scanners is the filtered backprojection (FBP) method ${ }^{1)}$. Statistical approaches for image reconstruction in PET have received considerable attention in recent years because of their

"All correspondence to Kenya Murase, PhD, Department of Radiology, Ehime University School of Medicine, Shitsukawa, Shigenobu-cho, Onsen-gun, Ehime-Pref. 791-02, Japan, Tel. : 089-964-5111 ext. 2104, Fax : 089-964-2902, e-mail : murase@dpc.ehime-u.ac.jp. 
potential for improving the image quality. Of those approaches, the maximum likelihoodexpectation maximization (ML-EM) method $^{2)}$ is a fundamental algorithm, which has been used as a basis in other statistical approaches. However, the ML-EM method has some drawbacks ${ }^{3)}$; the statistical noise, sharp boundaries or edges in the reconstructed images are greatly accentuated with increasing iterations. In order to overcome these problems, several statistical methods have also been proposed ${ }^{3)-5}$.

Magnetic resonance imaging (MRI) provides high-resolution morphological information, and this can be used to classify different tissues such as gray matter, white matter and extracerebral spaces ${ }^{6,7)}$. Then, it is expected that we can get better PET images by incorporating these informations into the image reconstruction process. There are several image reconstruction algorithms that utilize anatomical information as priors ${ }^{8), 99}$. However, some restrictive assumptions are necessary in these algorithms. For example, the tracer distribution within an MRI-defined tissue class is assumed to be homogeneous. Recently, one of the authors (YZ) has proposed a new approach with fewer restrictions, which we termed "Bayesian method with both gradient and tissue classification priors (BGT method)"*. In the present study, we evaluated the usefulness of this method using simulated PET projection data generated from an MRI-based three-dimensional (3-D) brain phantom including the effects of resolution, attenuation, scatter and Poisson noise.

\section{Materials and Methods}

\subsection{Background}

The task of PET image reconstruction is to estimate $\lambda\left(=\left\{\lambda_{k}\right\}\right)$ given $Y\left(=\left\{Y_{t}\right\}\right)$, where $\lambda_{k}$ is

* Zhang, Y., Ma, Y., Murase, K. and Evans, A.C., in preparation. the image value at pixel $k$, and $Y_{t}$ is the number of coincidences registered in detector pair $t$. In the ML-EM method ${ }^{2}$, the formula for updating estimates in the iterative process is

$$
\lambda_{k}{ }^{(i+1)}=\lambda_{k}{ }^{(i)} \cdot \sum_{t=1}^{T} \frac{Y_{t} a_{k t}}{\sum_{l=1}^{K} \lambda_{l}{ }^{(i)} a_{l t}}
$$

where $a_{k t}$ denotes the probability coefficients which give the probability that an event occurring at pixel $k(1, \ldots \ldots, K)$ is recorded in detector pair $t(1, \ldots \ldots, T) . \lambda_{k}{ }^{(i)}$ denotes the estimate of $\lambda_{k}$ at iteration $i$.

For reducing statistical noise and edge artifacts introduced when using the ML-EM method ${ }^{3)}$, Johnson et al. ${ }^{5)}$ and Chen et al. ${ }^{10)}$ have reported on a Bayesian model for PET image reconstruction. With their methods, statistical noise was effectively suppressed by means of a Poisson model for data acquisition and a Gibbs distribution used as the prior which incorporated the property of local continuity ${ }^{11)}$ (i.e., neighboring pixels within homogeneous regions have similar intensity levels). Furthermore, Johnson et al. ${ }^{5)}$ considered the boundaries within the image to be variable, and incorporated the boundaries into a Bayesian model using a so-called "line process". The line process regulates the degree of smoothing between neighboring pixels. In this paper, we will term the approach developed by Johnson et al. ${ }^{5)}$ "BAY method".

Chen et al. $^{10)}$ and Ouyang et al. ${ }^{12)}$ have expanded the approach of Johnson et al. ${ }^{5)}$, using structural images obtained from X-ray computed tomography (CT) or MRI as a prior information. In this approach, the "weighted line site method" ${ }^{12)}$ was introduced in order to selectively use the prior boundaries, because anatomical boundaries do not always coincide with or correspond to functional boundaries. The "weighted line site method" was based on the crosscorrela- 
tion between the anatomical and functional boundary maps ${ }^{12}$.

\subsection{BGT method}

The BGT method is an extension of the methods proposed by Johnson et al. ${ }^{5)}$ and Ouyang et al. ${ }^{12)}$, and utilizes not only anatomical information but also the tissue classification information obtained from MR images. Tissue classification probability images for gray matter (GM), white matter (WM), and cerebrospinal fluid (CSF) can be generated from segmented MR images by means of a tissue classification method ${ }^{6,7)}$.

For explanation of the BGT method, we will use Probability $[m]_{k}$ to denote the tissue classification probability image for the tissue type " $m$ " (GM, WM, CSF, etc.) at pixel $k$. First, we measured the apparent radioactivity ratio between GM and other tissues such as WM and CSF as follows :

$$
\begin{aligned}
& \text { apparent_ratio }[m]= \\
& \frac{\text { total_activity }[\mathrm{GM}] / \text { total_number }[\mathrm{GM}]}{\text { total_activity }[m] / \text { total_number }[m]}
\end{aligned}
$$

$$
\begin{aligned}
& \text { total_activity }[m] \\
& \quad=\sum_{k=1}^{K} \lambda_{k}^{(i)} \times \operatorname{Probability}[m]_{k} \\
& \text { total_number }[m] \\
& \quad=\sum_{k=1}^{K} \operatorname{Probability}[m]_{k}
\end{aligned}
$$

This apparent radioactivity ratio is generally smaller than the true ratio because of the partial volume effect. To compensate for the partial volume effect, we used the following algorithm to increase the apparent radioactivity ratio between GM and other tissues such as WM and CSF.

In order to modify the apparent radioactivity ratio, we introduced a new parameter $f(m)$, which is a pre-selected constant for increasing the apparent ratio. Typically, $f(\mathrm{GM})$ and $f(\mathrm{WM})$ were fixed to 1.0 and 2.0 , respectively. The detailed algorithm is as follows:

1. Measure apparent_ratio[m] using Eq.(2).

2. Calculate new_ratio $[m]$ as

$$
\begin{aligned}
& \text { new_ratio }[m] \\
& \quad=\text { apparent_ratio }[m] \times f(m)
\end{aligned}
$$

3. Calculate new total_activity $[m]$ denoted by new_total $[m]$ as new_total $[m]$

$=Q \times$ total_number $[m] /$ new_ratio $[m]$

where

$$
Q=\frac{\sum_{t=1}^{T} Y_{t}}{\sum_{m=1}^{M} \text { total_number }[m] / \text { new_ratio }[m]}
$$

where $M$ is the total number of tissu types.

4. Modify all pixel values as

$$
\begin{aligned}
& \lambda_{k}{ }^{(i+1)}=\lambda_{k}{ }^{(i)} \times \\
& \left(\sum_{m=1}^{M} \text { Probability }[m]_{k} \times \frac{\text { new_total }[m]}{\operatorname{total} \_\operatorname{activity}[m]}\right)
\end{aligned}
$$

where the superscript ( $i$ ) denotes the current estimate of $\lambda_{k}$. Note that Eq.(6) is obtained by solving the following equations.

$$
\left\{\begin{array}{l}
\text { new_ratio }[m]= \\
\frac{\text { new_total }[\mathrm{GM}] / \text { total_number }[\mathrm{GM}]}{\text { new_total }[m] / \text { total_number }[m]} \\
\sum_{m=1}^{M} \text { new_total }[m]=\sum_{t=1}^{T} Y_{t}
\end{array}\right.
$$

Subsequently, the cross-correlation between anatomical and enhanced functional boundary maps was incorporated into BAY using the weighted line site method ${ }^{12)}$. We started the reconstruction process with the BAY method ${ }^{5)}$, and typically after 7 iterations, we switched to the BGT method. 


\section{$2 \cdot 3$ Simulation study}

The reconstruction methods were tested with simulated PET projection data generated from an MRI-based 3-D PET brain phantom, including the effects of resolution, attenuation, scatter and Poisson noise added at the projection level ${ }^{13)}$. The relative intensities of pixel values in GM, WM and CSF were assigned as 4,1 and 0 , respectively.

\subsection{Comparison with FBP, ML-EM and} BAY methods

In order to validate the BGT method, we compared with the FBP ${ }^{1)}$, ML-EM ${ }^{2)}$ and $\mathrm{BAY}^{5)}$ methods. When using the FBP method, Hanning filter with a FWHM of $10 \mathrm{~mm}^{13)}$ was used. When using the BAY method, the Gibbs smoothness prior $(\sigma)^{5)}$ was fixed to 2.16 . When using the BGT method, the thresholds used in the weighted line site method $\left(\sigma_{\mathrm{F}, \min } \text { and } \sigma_{\mathrm{A}, \min }\right)^{12)}$ were fixed to 0.75 and 0.25 , respectively.

\section{$2 \cdot 5$ Evaluation}

The reconstructed images were evaluated in terms of root mean square distance (RMSD) from the true image, which is defined as

$$
\begin{aligned}
\operatorname{RMSD}= & \left(\sqrt{\sum_{k=1}^{N}\left(\lambda_{k}-t_{k}\right)^{2} / N} / t_{m}\right) \\
& \times 100(\%)
\end{aligned}
$$

where $t_{m}=\sum_{k=1}^{N} t_{k} / N, \lambda_{k}$ is the reconstructed image value at pixel $k, t_{k}$ the true image value at pixel $k$, and $N$ the total number of pixels.

\section{Results}

Figures 1 (a) and 2(a) show the PET images reconstructed using the FBP (upper left), MLEM (upper right), BAY (lower left) and BGT (lower right) methods. On the other hand, Figs. 1 (b) and 2(b) show the relationships between RMSD and iteration number in the images reconstructed using the above methods. Figures 1 and 2 are the cases when the total count in the true image is $98 \mathrm{k}$ and $980 \mathrm{k}$ counts, respectively.

As shown in Figs. 1(a) and 2(a), the PET images reconstructed by the BGT method were superior in quality to those obtained by the FBP, ML-EM and BAY methods. Table 1 summarizes the minimum RMSD in the images reconstructed by the FBP, ML-EM, BAY, and BGT method as a parameter of the total count. The BGT method improved RMSD by $39.6 \%, 33.5 \%$ and $33.0 \%$ on average, compared to the FBP, ML-EM and BAY method, respectively.

\section{Discussion}

The BGT method demonstrated here is an extension of the methods developed by Johnson et al. ${ }^{5)}$ and Ouyang et al. ${ }^{12)}$, in which the functional PET image gradient is enhanced in a modified updating scheme with the use of both the PET image and tissue classification probability images generated from segmented MR images. Several methods ${ }^{6,7)}$ have been developed and available for generating tissue classification probability images. The BGT method incorporates the tissue classification information into the image reconstruction process as a prior in a Bayesian model ${ }^{9,11)}$ in addition to anatomical information. In this respect, this method is different from other methods utilizing anatomical images such as CT or MR images ${ }^{8), 9)}$. Results from computer simulations showed that incorporation of both the anatomical and tissue classification information obtained from spatially correlated MRI data considerably improved the quality of PET images (Figs. 1 (a) and 2 (a)). The PET images reconstructed by this method also appear to be quantitatively more accurate than those obtained by the other methods (Table 1).

One attractive feature of the Bayesian reconstruction method is the ability to use a prior 
(a)

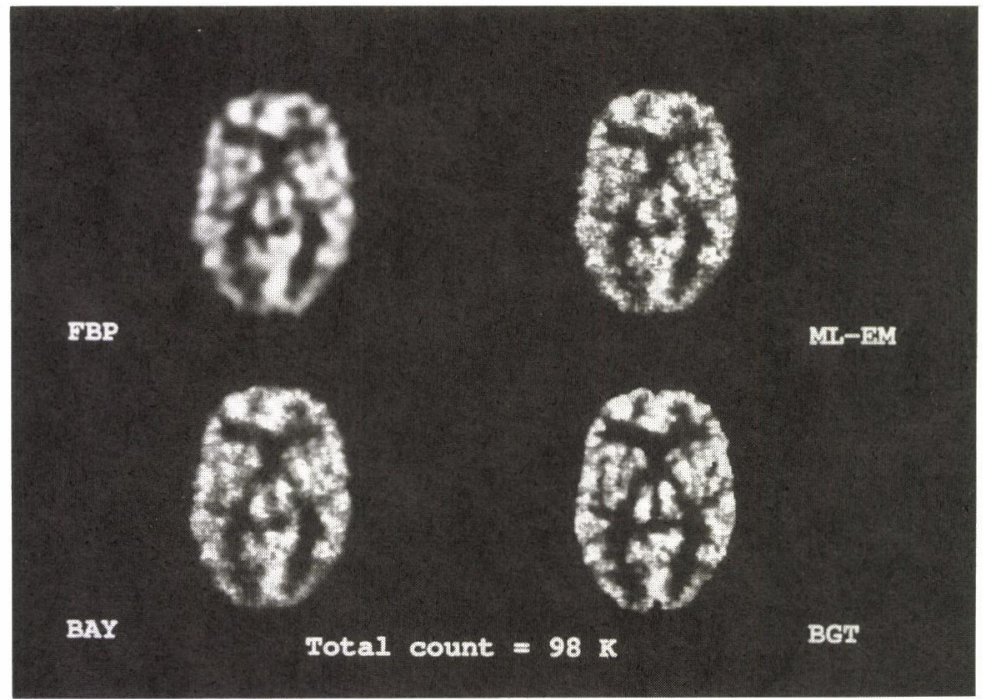

(b)

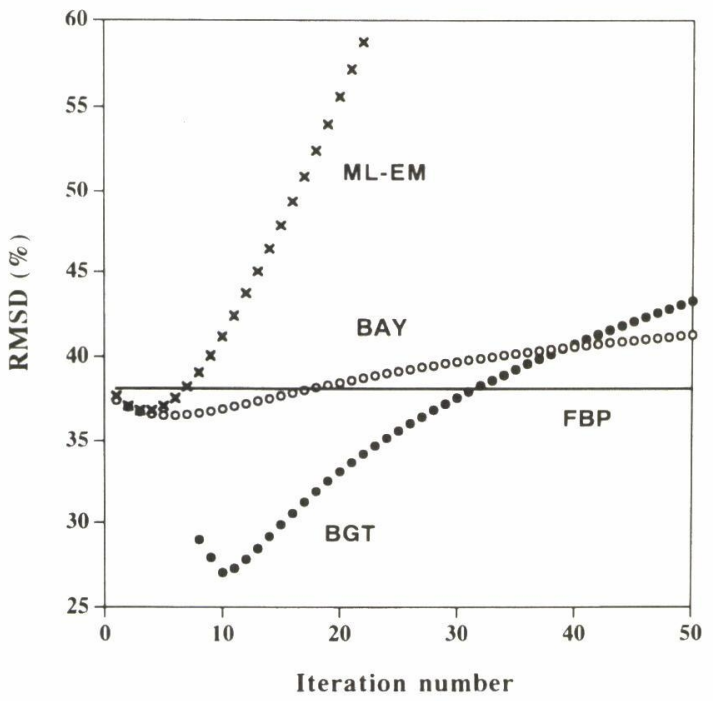

information to regulate the property of the posterior estimates such as statistical noise ${ }^{3)}$ and image contrast $^{9)}$. Anatomical boundaries can be introduced as a prior in the line process ${ }^{5}$ to enhance the spatial resolution and image contrast of the functional PET images. By 3-D image registration and sampling, it is possible to obtain the MR image that represents the anatomy of the corresponding PET image. However, because anatomical boundaries do not always coincide with or correspond to functional boundaries, direct super-
Fig. 1 (a) PET images reconstructed using the FBP (upper left), ML-EM (upper right), BAY (lower left) and BGT (lower right) methods when the total count in the true image is $98 \mathrm{k}$ counts.

(b) Relationship between RMSD and iteration number when the total count in the true image is $98 \mathrm{k}$ counts.

(- : FBP method, $\times$ : ML-EM method, $\bigcirc$ : BAY method, - BGT method)

position of anatomical boundaries onto PET images may produce artifacts. Then, Ouyang et al. ${ }^{12)}$ introduced the weighted line site method in order to reduce the artifacts due to misalignment between anatomical and functional image boundaries. The weighted line site method is based on the cross-correlation between anatomical and functional boundaries.

Although we adopted the weighted line site method in the BGT method to reduce the artifacts due to the inadequate registration between the 
(a)

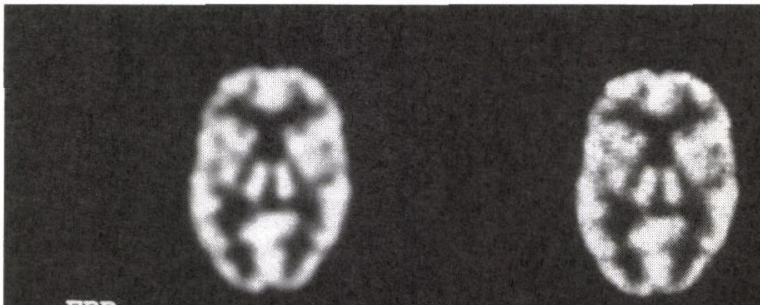

FBP

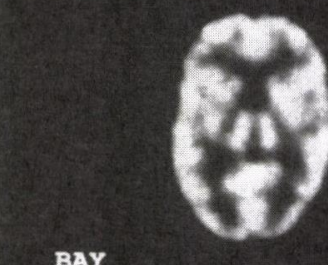

Total count $=980 \mathrm{~K}$

BGI

(b)

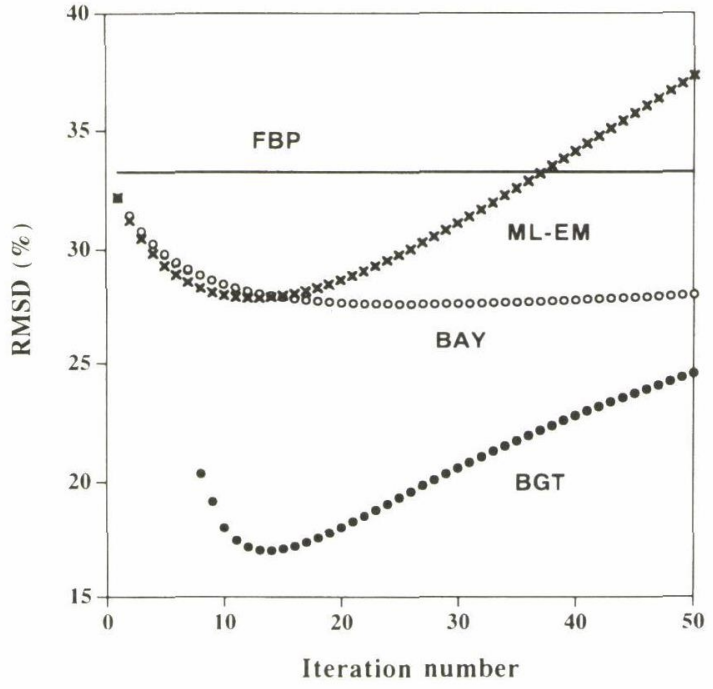

Table 1 Minimum RMSD(\%) in the images reconstructed by the FBP, ML-EM, BAY, and BGT method as a parameter of the total count in the true image

\begin{tabular}{ccccc}
\hline \multirow{2}{*}{$\begin{array}{c}\text { Total } \\
\text { count } \\
\text { counts) }\end{array}$} & \multicolumn{4}{c}{ Image reconstruction method } \\
\cline { 2 - 5 } & FBP & ML-EM & BAY & BGT \\
\hline $98 \mathrm{k}$ & 38.1 & 36.9 & 36.6 & 27.0 \\
$245 \mathrm{k}$ & 34.9 & 32.3 & 32.4 & 22.5 \\
$490 \mathrm{k}$ & 35.3 & 31.1 & 30.7 & 19.4 \\
$980 \mathrm{k}$ & 33.3 & 27.9 & 27.6 & 17.0 \\
\hline
\end{tabular}

Fig. 2 (a) PET images reconstructed using the FBP (upper left), ML-EM (upper right), BAY (lower left) and BGT (lower right) methods when the total count in the true image is $980 \mathrm{k}$ counts.

(b) Relationship between RMSD and iteration number when the total count in the true image is $980 \mathrm{k}$ counts.

$(-$ : FBP method, $x$ : ML-EM method, $\bigcirc$ : BAY method, : BGT method)

anatomical MR image and the functional PET image, more accurate registration between them is needed. Current intermodality registration techniques are adequate for some image analysis and visualization $^{14)}$. However, they are not always adequate for the purpose of multimodal image reconstruction.

Although we showed that we can improve PET images using anatomical and tissue classification information obtained from MR images, we realize 
that successful application of the present method to routine clinical studies will require further progress of the following items: (i) segmentation of MR images and (ii) registration of the MR images with the PET images. Current techniques for segmenting the anatomical brain images provided by MRI need to be improved in order to provide more precise anatomical prior information. As mentioned above, more accurate registration of the prior anatomical MR and PET images is also needed. In addition to further progress of the above items, the detailed analyses concerning their effect on the accuracy of the present method are needed and are currently in progress. Although we investigated the usefulness of the present method using a relatively simple brain phantom in this study, further studies using a more complicated brain phantom ${ }^{15}$ ) and/or real data are considered to be necessary to establish the clinical usefulness of the present method. These studies are also currently being carried out.

In conclusion, our preliminary results suggest that incorporation of both anatomical and tissue classification information into image reconstruction process is promising for improving the qualitative and quantitative accuracy of PET images. Although we focused on PET in this study, the present method could be applied to single photon emission computed tomography (SPECT) with minor modification.

\section{References}

1) Shepp, L. A. and Logan, B. F.: The Fourier reconstruction of a head section, IEEE Trans. Nucl. Sci., NS-21, 21-43 (1974)

2) Shepp, L. A. and Vardi, Y.: Maximum likelihood reconstruction for emission tomography, IEEE Trans. Med. Imag., MI-1, 113-122 (1982)

3) Snyder, D. L., Miller, M. I., Thomas, L. J. and Polite, D. G. : Noise and edge artifacts in maximum-likelihood reconstruction for emission tomography, ibid., MI-6, 228-238 (1987)

4) Levitan, E. and Herman, G. T. : A maximum a posterior probability expectation maximization algorithm for image reconstruction in emission tomography, ibid., MI-6, 185-192 (1987)

5) Johnson, V. E., Wong, W. H., Hu, X. and Chen, C. T.: Image restoration using Gibbs priors: boundary modeling, treatment of blurring, and selection of hyperparameter, IEEE Trans. Pattern Anal. Mach. Intell., 13, 413-425 (1991)

6) Kamber, M., Shinghal, R., Collins, D. L., Francis, G. S. and Evans, A. C. : Model-based 3-D segmentation of multiple sclerosis lesions in magnetic resonance brain images, IEEE Trans. Med. Imag., 14, 442-453 (1995)

7) Bullmore, E., Brammer, M., Rouleau, G., Evevitt, B., Simmons, A., Sharma, T., Frangou, S., Murray, R. and Dunn, G. : Computerized brain tissue classification of magnetic resonance images: a new approach to the problem of partial volume artifacts, Neuroimage, 2, 133-147 (1995)

8) Leathy, R. and Yan, X.: Incorporation of anatomical MR data for improved functional imaging with PET, Information Processing in Medical Imaging, 12th International Conference, (Colchester, A. C. F. and Hawkes, D. J. eds.), pp. 105120, Springer-Verlag, London (1991)

9) Gindi, G., Lee, M., Rangarajan, A. and Zubal, I. G. : Bayesian reconstruction of functional images using anatomical information as priors, IEEE Trans. Med. Imag., 12, 670-680 (1993)

10) Chen, C. T., Ouyang, X., Wong, W. H., Hu, X., Johnson, V. E., Ordonez, C. and Mets, C. E.: Sensor fusion in image reconstruction, IEEE Trans. Nucl. Sci., 38, 687-692 (1991)

11) Geman, S., and Geman, D. : Stochastic relaxation, Gibbs distributions, and the Bayesian restoration of images, IEEE Trans. Pattern Anal. Mach. Intell., PAMI-6, 721-741 (1984)

12) Ouyang, X., Wong, W. H., Johnson, V. E., Hu, X. and Chen, C. T.: Incorporation of correlated structural images in PET image reconstruction, IEEE Trans. Med. Imag., 13, 627-640 (1994)

13) Ma, Y., Kamber, M. and Evans, A. C. : 3-D simulation of PET brain images using segmented MRI and positron tomograph characteristics, 14th Ann. Int. Conf. IEEE-EMBS, pp. 141-145, Rennes, France (1992)

14) Evans, A., Marret, S., Torrescorzo, J., Ku, S. and Collins, L. : MRI-PET correlation in three dimensions using a volume of interest (VOI) atlas, $J$. Cereb. Blood Flow Metab., 11, 169-178 (1991)

15) Murase, K., Zhang, Y., Ma, Y. and Evans, A. C. : Usefulness of a newly-developed MRIconstrained PET image reconstruction method, J. Nucl. Med., 37, 223 P (1996) (Abstract) 